\title{
Cardiovascular, thromboembolic and renal outcomes in IgA vasculitis (Henoch-Schönlein purpura): a retrospective cohort study using routinely collected primary care data
}

\author{
Alexander Tracy, ${ }^{1}$ Anuradhaa Subramanian, ${ }^{2}$ Nicola J Adderley, ${ }^{2}$ Paul Cockwell, ${ }^{3}$ \\ Charles Ferro, ${ }^{3}$ Simon Ball, ${ }^{3}$ Lorraine Harper, ${ }^{1}$ Krishnarajah Nirantharakumar $^{2}$
}

\section{Handling editor Josef S Smolen}

- Additional material is published online only. To view please visit the journal online (http://dx.doi.org/10.1136/ annrheumdis-2018-214142)

${ }^{1}$ Centre for Translational Inflammation Research, Institute of Clinical Sciences, University of Birmingham, Birmingham, UK ${ }^{2}$ Institute of Applied Health Research, University of Birmingham, Birmingham, UK ${ }^{3}$ Department of Renal Medicine, University Hospitals Birmingham NHS Trust, Birmingham, UK

\section{Correspondence to}

Dr Lorraine Harper, Centre for Translational Inflammation Research, Institute of Clinical Sciences, University of Birmingham, Birmingham B15 2WB, UK; l.harper@bham.ac.uk

$\mathrm{LH}$ and $\mathrm{KN}$ contributed equally.

Received 18 July 2018 Revised 30 October 2018 Accepted 1 November 2018 Published Online First 28 November 2018
Check for updates

(c) Author(s) (or their employer(s)) 2019. No commercial re-use. See rights and permissions. Published by BMJ.

To cite: Tracy $A$,

Subramanian A, Adderley NJ, et al. Ann Rheum Dis

2019;78:261-269

\section{ABSTRACT}

Background IgA vasculitis (IgAV, Henoch-Schönlein purpura) is a small-vessel vasculitis most common in children but also occurring in adults. Case series have suggested that IgAV may be associated with cardiovascular disease and venous thromboembolism, but this has not been evaluated in population-based studies. Renal disease and hypertension are possible complications of the disease with unknown incidence. Methods Using a large UK primary care database, we conducted an open retrospective matched cohort study of cardiovascular, venous thrombotic and renal outcomes in adult-onset and childhood-onset IgAV. Control participants were selected at a 2:1 ratio, matched for age and sex. Adjusted HRs (aHRs) were calculated using Cox proportional hazards models.

Results 2828 patients with adult-onset IgAV and 10405 patients with childhood-onset IgAV were compared with age-matched and sex-matched controls. There was significantly increased risk of hypertension (adult-onset aHR 1.42, 95\% Cl 1.19 to $1.70, \mathrm{p}<$ 0.001; childhood-onset aHR $1.52,95 \% \mathrm{Cl} 1.22$ to $1.89, p<0.001)$ and stage G3-G5 chronic kidney disease (adult-onset aHR 1.54, 95\% Cl 1.23 to 1.93, $\mathrm{p}<0.001$; childhood-onset aHR $1.89,95 \% \mathrm{Cl} 1.16$ to $3.07, p=0.010)$. There was no evidence of association with ischaemic heart disease, cerebrovascular disease or venous thromboembolism. All-cause mortality was increased in the adult-onset IgAV cohort compared with controls (aHR 1.27, 95\% Cl 1.07 to $1.50, p=0.006$ ).

Conclusions Patients with IgAV are at increased risk of hypertension and chronic kidney disease (CKD) compared with individuals without IgAV; analysis restricted to adult-onset IgAV patients showed increased mortality. Appropriate surveillance and risk factor modification could improve long-term outcomes in these patients.

\section{INTRODUCTION}

IgA vasculitis (IgAV), also termed Henoch-Schönlein purpura, is a small-vessel vasculitis most frequently affecting children. ${ }^{1}$ IgAV is the most common childhood vasculitis in the UK, with estimated annual incidence of 20/100 000 children under the age of 17 years according to the largest regional study. ${ }^{2}$ However, incidence rates vary widely between study populations, and, furthermore, these may represent underestimates of true incidence. ${ }^{3}$ The epidemiology of adult-onset IgAV is

\section{Key messages}

What is already known about this subject?

- IgA vasculitis (IgAV) is recognised to occur in both children and adults; however, the incidence and prevalence, especially in adults, is unknown in large populations based in primary care.

- IgAV is associated with long-term complications including chronic kidney disease.

- Small case series have previously suggested a predisposition to ischaemic heart disease and venous thromboembolism in some patients.

What does this study add?

- The risk of hypertension and CKD is significantly increased in adult-onset and childhood-onset IgAV compared with the general population.

- IgAV is not significantly associated with ischaemic heart disease or venous thromboembolism in this study.

- The young age of most patients and short follow-up in this study mean longer follow-up is required to address the risk of ischaemic heart disease.

How might this impact on clinical practice or future developments?

- Clinicians looking after patients who have had IgAV should routinely monitor for hypertension and CKD

less well-studied, but hospital-based studies indicate an estimated annual incidence of 0.8-1.8/100 000 population.

IgAV may be complicated by glomerulonephritis ${ }^{4}$ and it is thought that adult-onset IgAV is associated with increased risk and severity of renal involvement compared with childhood disease. ${ }^{5} 6$ However, long-term health outcomes of adult-onset IgAV are not well characterised. Most evidence regarding complications of IgAV in adults derives from case reports and case series ${ }^{7}$; there is need for controlled epidemiological studies to address this question.

Other outcomes associated with IgAV are unknown. Multiple case reports have raised the possibility of associations between IgAV and venous thromboembolism (VTE), hypertension and 
ischaemic heart disease (IHD) in both children and adults. ${ }^{8-19}$ However, many cases involved patients with additional risk factors, making the role of IgAV unclear. To date, the incidence of these outcomes has not been examined in a large cohort study. Furthermore, there is emerging evidence that patients with other vasculitides have increased incidence of cardiovascular disease $^{2021}$ and receive inadequate management of cardiovascular risk. ${ }^{22}$

This study aims to calculate incidence of IgAV in adults and children, and to quantify risk of important complications in adult and childhood-onset disease, in particular, risk of cardiovascular, thromboembolic and renal outcomes. These data will facilitate prognostication in such patients, thus informing strategies for surveillance and risk factor modification in routine care.

\section{METHODS}

\section{Study design}

Incidence and prevalence of IgAV

To calculate IgAV incidence, annual cohort studies were performed between 1 January 2005 and 31 December 2016. To estimate prevalence, sequential cross-sectional studies were carried out on 1 January each calendar year from 2005 to 2016.

\section{Chronic outcomes}

An open retrospective matched cohort study was performed to compare long-term cardiovascular, venous thromboembolic and renal outcomes in adults and children diagnosed with IgAV and randomly selected age-matched and sex-matched controls without a diagnosis. The study period was 1 January 1995 to 15 May 2017.

\section{Data source}

Data were extracted from The Health Improvement Network (THIN) database, which comprises anonymised medical records for 3.6 million active patients from $>675$ general practices, as previously reported. ${ }^{23} 24$ Patient data are derived from practices using Vision electronic medical record software, which stores information in a hierarchical system of clinical (Read) codes. ${ }^{25}$ THIN includes information on patient demographics, diagnoses, prescriptions and investigations. THIN has previously been validated for studies of cardiovascular and renal outcomes, ${ }^{26} 27$ and for studies of VTE risk. ${ }^{28}$ It is broadly representative of the UK population in terms of demographics, disease prevalence and mortality rates. ${ }^{23}$ To maximise data quality, general practices were only included in this study from the latest of 1 year after they began using Vision software and 1 year after their acceptable mortality recording date. ${ }^{24}$ Diagnosis was based on Read codes which registered a clinical diagnosis of Henoch-Schönlein purpura, and not IgA deposition within tissues.

\section{Study population}

Incidence and prevalence of IgAV

Adults and children with no record of a IgAV diagnosis at the beginning of each 1-year study period were included in the annual incidence cohorts. The eligible populations were followed from 1 January every year until the earliest of the following dates: IgAV diagnosis, patient left the practice, death or 31 December of that year. Annual prevalence is reported per 100000 population.

\section{Chronic outcomes}

In the adult-onset IgAV cohort, patients were eligible for inclusion if they had a clinical code for IgAV recorded at age $\geq 16$ years. Inclusion in the childhood-onset IgAV cohort was restricted to patients with recorded diagnosis of IgAV before the age of 16 years. For each patient with IgAV, two age-matched and sex-matched control patients were randomly selected from a pool of eligible controls. All patients were required to be registered with their general practice for at least 1 year before study entry.

Index date in the exposed group was the date of first documentation of IgAV after study entry for incident cases (newly diagnosed patients) or date of study entry for prevalent cases (patients with an existing diagnosis). To avoid immortal time bias, ${ }^{29}$ controls were assigned the same index date as their corresponding exposed patient. Participants were followed up until the earliest of the following dates: outcome event, death, patient left practice, practice stopped contributing to the database and study end.

\section{Outcomes}

In patients with adult-onset IgAV, primary outcomes were IHD, VTE, stroke/transient ischaemic attack (TIA), hypertension, stage G3-G5 CKD and all-cause mortality. IHD, VTE, stroke/TIA and hypertension were defined by clinical (Read) codes; stage G3-G5 CKD was defined by new-onset estimated glomerular filtration rate (eGFR) $<60 \mathrm{~mL} / \mathrm{min} / 1.73 \mathrm{~m}^{2}$ on two consecutive measurements separated by at least 90 days. ${ }^{30}$ Clinical codes were selected based on quality and outcomes framework (QOF) business rules and previously published studies. $^{3132}$

For hypertension, a sensitivity analysis was performed using a lag period of 1 year to test whether this represented a chronic outcome or was solely related to the acute illness.

In the childhood-onset IgAV cohort, primary outcomes were hypertension, VTE and CKD. IHD, stroke/TIA and mortality were not studied in this cohort due to low incidence in this age group and short follow-up period.

\section{Analysis}

Annual incidence rates (IRs) of IgAV were calculated by dividing the number of newly diagnosed IgAV patients by person-years at risk for adults and children separately.

Cox proportional hazards models were used to calculate crude HRs and adjusted HRs (aHR) for each outcome in IgAV compared with controls. Breslow's method was used to handle tied survival times where required. All models were adjusted for the following covariates: age, sex, body mass index (BMI) category, Townsend deprivation quintile and smoking status. Additionally, the models for IHD and stroke/TIA were adjusted for baseline diabetes, hypertension and lipid-lowering drug prescription; the model for hypertension was adjusted for baseline diabetes and lipid-lowering drug prescription; the model for CKD was adjusted for baseline diabetes and hypertension; and the model for all-cause mortality was adjusted for lipid-lowering drug prescription and Charlson comorbidity index (CCI). For childhood-onset IgAV, BMI category, lipid-lowering drug prescription and smoking status were not included in the models. BMI recorded closest to index date was categorised as ' $<25 \mathrm{~kg} / \mathrm{m}^{2}$, ' $25-30 \mathrm{~kg} / \mathrm{m}^{2}$ ' (overweight) and ' $>30 \mathrm{~kg} / \mathrm{m}^{2}$ ' (obesity); smoking status was categorised as 'smoker', 'ex-smoker' and 'non-smoker'. Social deprivation was categorised according to Townsend deprivation quintile. ${ }^{33}$ 
CCI was categorised as ' 0 ', ' 1 ', ' 2 ' or ' $>2$ '. ${ }^{34}$ Separate categories were created for missing data, which were included in the regression analyses.

Baseline renal function was not adjusted for in the primary analysis due to limited availability of creatinine measurements before the index date. A sensitivity analysis was performed in which all models were adjusted for baseline eGFR.

For the adult-onset and childhood-onset studies, all patients without a record of the outcome under study at baseline were included in the primary analysis. For the CKD study, primary analysis included only patients with an eGFR $>60 \mathrm{~mL} /$ $\min / 1.73 \mathrm{~m}^{2}$ at baseline. Sensitivity analyses were performed in which (1) patients with missing baseline eGFR values were included and categorised as having normal renal function and (2) all patients were included regardless of baseline eGFR, assuming that baseline eGFR might reflect transient residual renal impairment.
To ensure that results are applicable to disease of adult onset, a sensitivity analysis was performed using incident (newly diagnosed, definite adult-onset) adult IgAV cases only.

Analyses were performed using STATA V.14.0. Statistical significance was set at $\mathrm{p}<0.05$.

\section{RESULTS}

\section{Incidence of IgAV in adults and children}

Between 2005 and 2016, incidence of childhood-onset IgAV was 27.22 per 100000 person-years; incidence of adult-onset IgAV was 2.20 per 100000 person-years (figure 1; baseline characteristics are summarised in online supplementary table S2. Mean (SD) age at diagnosis was 6.68 years (3.41) years for children and 38.1 (18.8) years for adults. While IgAV incidence remained stable, prevalence of both adult-onset and childhood-onset IgAV increased over the study period. 
Between 2005 and 2016, prevalence of adult-onset IgAV increased from 34 to 44 per 100000 population; prevalence of childhood-onset IgAV increased from 621 to 846 per 100 000 population.

\section{Outcomes in adult-onset IgAV}

\section{Baseline characteristics}

There were 2828 patients with adult-onset IgAV and 5655 controls. Median (IQR) follow-up was similar in both cohorts: 4.91 2.07-9.08 years in the IgAV cohort and 4.99 2.18-9.05 years in the control cohort.
Mean age at study entry was 43 years in both exposed and unexposed cohorts. Both cohorts had $48.4 \%$ males. At baseline renal impairment (eGFR $<60 \mathrm{~mL} / \mathrm{min} / 1.73 \mathrm{~m}^{2}$ ) was more common in patients with IgAV (6.86\% vs $4.05 \%$ in unexposed). Similar differences between those with and without IgAV were observed for hypertension (18.5\% vs $13.0 \%)$, diabetes mellitus (5.3\% vs $3.7 \%)$, VTE $(1.8 \%$ vs $1.1 \%)$, lipid-lowering drug prescription (11.1\% vs $5.0 \%)$ and CCI $(15.28 \%$ vs $7.35 \%$ with $\geq 2$ comorbidities). Patients with IgAV were less likely to be current smokers $18.5 \%$ versus $22.8 \%$. The cohorts were similar with respect to BMI and Townsend deprivation quintile (table 1).

Table 1 Baseline characteristics of the adult-onset and childhood-onset $\lg A$ vasculitis (IgAV) cohorts with corresponding controls. adult-onset patients include all incident and prevalent IgAV cases with a date of diagnosis after the age of 16 years

\begin{tabular}{|c|c|c|c|c|}
\hline \multicolumn{5}{|c|}{ Baseline characteristics (SD or percentage) } \\
\hline & \multicolumn{2}{|l|}{ Adult-onset IgAV } & \multicolumn{2}{|c|}{ Childhood-onset IgAV } \\
\hline & Exposed & Unexposed & Exposed & Unexposed \\
\hline Median follow-up period (years) & 4.91 (IQR 2.07-9.08) & 4.99 (IQR 2.18-9.05) & 4.86 (IQR 2.06-9.08) & 4.98 (IQR 2.17-9.86) \\
\hline Mean age at study entry (years)* & $43.33(18.8)$ & $43.33(18.7)$ & $17.57(13.12)$ & $17.59(13.13)$ \\
\hline Mean age at IgAV diagnosis & $38.09(18.8)$ & N/A & $6.68(3.41)$ & $\mathrm{N} / \mathrm{A}$ \\
\hline Mean body mass index & $26.9(6.1)$ & $26.2(5.5)$ & N/A & $\mathrm{N} / \mathrm{A}$ \\
\hline \multicolumn{5}{|l|}{ Smoking status } \\
\hline Current smoker & $522(18.5 \%)$ & $1289(22.8 \%)$ & $1178(11.32 \%)$ & $2102(10.10 \%)$ \\
\hline Ex-smoker & $525(18.6 \%)$ & $845(14.9 \%)$ & $528(5.07 \%)$ & $904(4.34 \%)$ \\
\hline Non-smoker & $1525(53.9 \%)$ & $2825(50.0 \%)$ & $2751(26.44 \%)$ & $5427(26.08 \%)$ \\
\hline VTE & $52(1.8 \%)$ & $61(1.1 \%)$ & $29(0.28 \%)$ & $37(0.18 \%)$ \\
\hline IHD & $139(4.9 \%)$ & $220(3.9 \%)$ & $\mathrm{N} / \mathrm{A}$ & $\mathrm{N} / \mathrm{A}$ \\
\hline Stroke and TIA & $68(2.4 \%)$ & $107(1.9 \%)$ & N/A & $N / A$ \\
\hline \multicolumn{5}{|l|}{ eGFR category } \\
\hline$>90 \mathrm{~mL} / \mathrm{min}$ per $1.73 \mathrm{~m}^{2}$ & $639(22.60 \%)$ & $856(15.14 \%)$ & $116(0.56 \%)$ & $100(0.96 \%)$ \\
\hline $60-90 \mathrm{~mL} / \mathrm{min}$ per $1.73 \mathrm{~m}^{2}$ & $697(24.65 \%)$ & $1160(20.51 \%)$ & $121(0.58 \%)$ & $157(1.51 \%)$ \\
\hline $30-59 \mathrm{~mL} / \mathrm{min}$ per $1.73 \mathrm{~m}^{2}$ & $164(5.80 \%)$ & $209(3.70 \%)$ & $1339(6.43 \%)$ & $707(6.79 \%)$ \\
\hline$<30 \mathrm{~mL} / \mathrm{min}$ per $1.73 \mathrm{~m}^{2}$ & $30(1.06$ & $20(0.35 \%)$ & $1358(6.53 \%)$ & $642(6.17 \%)$ \\
\hline N/A & $1298(45.90 \%)$ & $3410(60.30 \%)$ & $17876(85.90 \%)$ & $8799(84.57 \%)$ \\
\hline Lipid-regulating medication use & $313(11.1 \%)$ & $506(5.0 \%)$ & N/A & $\mathrm{N} / \mathrm{A}$ \\
\hline Current contraceptive uset & $336(23.1 \%)$ & $572(19.6 \%)$ & $799(16.44 \%)$ & $1,432(14.73 \%)$ \\
\hline \multicolumn{5}{|l|}{ Townsend deprivation quintile } \\
\hline \multicolumn{5}{|l|}{ Charlson comorbidity index } \\
\hline 0 & $1806(63.86 \%)$ & $4176(73.85 \%)$ & $\mathrm{N} / \mathrm{A}$ & $\mathrm{N} / \mathrm{A}$ \\
\hline 1 & $590(20.86 \%)$ & $1063(18.80 \%)$ & N/A & $\mathrm{N} / \mathrm{A}$ \\
\hline 2 & $259(9.16 \%)$ & $258(4.56 \%)$ & N/A & $\mathrm{N} / \mathrm{A}$ \\
\hline$>2$ & $173(6.12 \%)$ & $158(2.79 \%)$ & N/A & $\mathrm{N} / \mathrm{A}$ \\
\hline
\end{tabular}

Childhood-onset patients include all incident and prevalent IgAV cases with a date of diagnosis before the age of 16 years. Controls were age-matched and sex-matched in a 2:1 ratio.

${ }^{*}$ Note that many patients had a IgAV diagnosis prior to study entry—prevalent cases. eGFR,estimated glomerular filtration rate; IHD, ischaemic heart disease; N/A, not available; VTE, venousthromboembolism.

tCurrent contraceptive use percentage reported for females only. 
Table 2 Summary of primary outcomes in adult-onset IgA vasculitis (IgAV) cases and corresponding controls

\begin{tabular}{|c|c|c|c|c|c|c|c|c|c|c|c|c|}
\hline & \multicolumn{2}{|c|}{ Hypertension } & \multicolumn{2}{|c|}{$\begin{array}{l}\text { Ischaemic heart } \\
\text { disease }\end{array}$} & \multicolumn{2}{|c|}{ Stroke/TIA } & \multicolumn{2}{|c|}{$\begin{array}{l}\text { Venous } \\
\text { thromboembolism }\end{array}$} & \multicolumn{2}{|c|}{$\begin{array}{l}\text { Chronic kidney } \\
\text { disease }\end{array}$} & \multicolumn{2}{|c|}{ All-cause mortality } \\
\hline & $\operatorname{IgAV}$ & Control & $\operatorname{IgAV}$ & Control & $\operatorname{IgAV}$ & Control & $\operatorname{Ig} A V$ & Control & $\operatorname{IgAV}$ & Control & $\operatorname{IgAV}$ & Control \\
\hline Patients, $\mathrm{n}$ & 2305 & 4921 & 2689 & 5435 & 2760 & 5548 & 2776 & 5594 & 2487 & 5225 & 2828 & 5655 \\
\hline $\begin{array}{l}\text { Numbers of } \\
\text { outcomes }\end{array}$ & 196 & 315 & 53 & 104 & 68 & 107 & 28 & 46 & 134 & 185 & 238 & 348 \\
\hline Person-years & 12847.60 & 28174.46 & 15974 & 32542.38 & 16552.39 & 33468.58 & 16771.07 & 33813.95 & 15359.92 & 32167.31 & 17085.13 & 34391.52 \\
\hline $\begin{array}{l}\text { Incidence rate (per } \\
1000 \text { person-years) }\end{array}$ & 15.26 & 11.18 & 3.32 & 3.20 & 3.14 & 3.26 & 1.67 & 1.36 & 8.72 & 5.75 & 13.93 & 10.12 \\
\hline Crude HR $(95 \% \mathrm{Cl})$ & \multicolumn{2}{|c|}{$1.36(1.14-1.63)$} & \multicolumn{2}{|c|}{$1.04(0.75-1.44)$} & \multicolumn{2}{|c|}{$0.96(0.69-1.34)$} & \multicolumn{2}{|c|}{$1.22(0.76-1.96)$} & \multicolumn{2}{|c|}{$1.52(1.22-1.90)$} & \multicolumn{2}{|c|}{$1.37(1.17-1.62)$} \\
\hline$P$ values & \multicolumn{2}{|l|}{0.001} & \multicolumn{2}{|l|}{0.829} & \multicolumn{2}{|l|}{0.814} & \multicolumn{2}{|l|}{0.401} & \multicolumn{2}{|l|}{$<0.001$} & \multicolumn{2}{|l|}{$<0.001$} \\
\hline Adjusted HR (95\% CI) & \multicolumn{2}{|c|}{$1.42(1.19-1.70)$} & \multicolumn{2}{|c|}{$1.08(0.77-1.52)$} & \multicolumn{2}{|c|}{$0.95(0.68-1.32)$} & \multicolumn{2}{|c|}{$1.21(0.76-1.95)$} & \multicolumn{2}{|c|}{$1.54(1.23-1.93)$} & \multicolumn{2}{|c|}{$1.27(1.07-1.50)$} \\
\hline$P$ values & \multicolumn{2}{|l|}{$<0.001$} & \multicolumn{2}{|l|}{0.637} & \multicolumn{2}{|l|}{0.758} & \multicolumn{2}{|l|}{0.424} & \multicolumn{2}{|l|}{$<0.001$} & \multicolumn{2}{|l|}{0.006} \\
\hline
\end{tabular}

\section{Hypertension}

In total, $196 \mathrm{IgAV}$ patients (6.93\%) received a diagnosis of hypertension compared with 315 (5.57\%) controls (table 2); incidence was 15.26 and 11.18 per 1000 person-years, respectively: aHR 1.42 (95\% CI 1.19 to 1.70). Cumulative hazard curves are shown in figure 2 . Results were robust in two sensitivity analyses restricting outcome to hypertension diagnosed at least 1 year after the index date and restricting to incident IgAV cases and their matched controls (online supplementary table S2).

\section{Ischaemic heart disease and cerebrovascular disease}

In total, 53 patients (1.87\%) with adult-onset IgAV and 104 $(1.84 \%)$ controls were diagnosed with IHD, corresponding to IRs of 3.32 and 3.20 per 1000 person-years, respectively. There was no evidence of association between IgAV and risk of IHD (aHR 1.08 , 95\% CI 0.77 to 1.52 ). Also, 52 patients with IgAV $(1.84 \%)$ and 109 controls (1.93\%) experienced a stroke/TIA, with IRs of 3.14 and 3.26 per 1000 person-years, respectively. There was no evidence of association between IgAV and risk of stroke/TIA (aHR $0.95,95 \%$ CI 0.68 to 1.32 ).

\section{Venous thromboembolism}

In total, 28 patients with adult-onset $\operatorname{IgAV}(0.99 \%)$ and 46 controls $(0.81 \%)$ were coded with a VTE event; incidence was 1.67 and 1.36 per 1000 person-years, respectively. Crude and adjusted HRs were not statistically significant: aHR 1.21 (95\% CI 0.76 to 1.95$)$.

\section{Chronic kidney disease}

There were 134 incident cases of CKD stages G3-5 (5.11\%) in the adult-onset IgAV cohort compared with 185 (3.42\%) in controls; incidence was 8.72 and 5.75 per 1000 person-years, respectively: aHR 1.54 (95\% CI 1.23 to 1.93 ). This association remained significant in all sensitivity analyses, including adjustment for baseline eGFR (online supplementary table S2).

\section{All-cause mortality}

There were 238 deaths $(8.42 \%)$ in the adult-onset IgAV cohort and 348 (6.15\%) in the control cohort, corresponding to mortality rates of 13.93 and 10.12 per 1000 person-years, respectively. In the primary analysis, all-cause mortality was significantly increased in the adult-onset IgAV cohort compared with controls: aHR 1.27 (95\% CI 1.07 to 1.50$)$. However, in a sensitivity analysis using incident cases and their controls only, the effect was not statistically significant (aHR 1.09, 95\% CI 0.83 to 1.42$)$.
Adjusting for baseline eGFR did not affect the results for any outcome (online supplementary table S3).

\section{Outcomes in childhood-onset IgAV}

\section{Baseline characteristics}

In total, 10405 patients with incident or prevalent childhood-onset IgAV were identified and matched to 20810 controls without IgAV. Mean (SD) age at diagnosis for the IgAV cohort was 6.68 (3.4); mean age at study entry was 17.6 (13.1) years and $53 \%$ were male in both cohorts. Median (IQR) follow-up was similar: $4.862 .06-9.08$ years in the IgAV cohort and 4.98 2.17-9.86 years in controls. At baseline, more patients with IgAV had hypertension $1.78 \%$ versus $1.13 \%$ and VTE $0.28 \%$ versus $0.18 \%$ compared with controls (table 1 ). The cohorts were also similar with respect to baseline renal function, Townsend deprivation quintile and smoking status.

\section{Hypertension}

In total, 139 patients with IgAV (1.34\%) and 193 controls (0.93\%) had hypertension (table 3); incidence was 2.29 and 1.57 per 1000 person-years, respectively: aHR 1.52 (95\% CI 1.22 to 1.89 ). This association remained significant in all of the sensitivity analyses performed (online supplementary table S3). Cumulative hazard curves are shown in figure 3.

\section{Venous thromboembolism}

In the childhood-onset IgAV cohort, 25 patients (0.24\%) experienced VTE compared with 46 controls $(0.22 \%)$; incidence was 0.40 and 0.37 per 1000 person-years, respectively. There was no evidence of association between childhood-onset IgAV and VTE (aHR $1.10,95 \%$ CI 0.68 to 1.79 ).

\section{Chronic kidney disease}

There were 32 incident cases of CKD $(0.31 \%)$ in patients with IgAV compared with $34(0.16 \%)$ in the controls; incidence was 0.51 and 0.27 per 1000 person-years, respectively: aHR 1.89 (95\%CI 1.16 to 3.07 ; see online supplementary table S3).

\section{DISCUSSION}

In this population-based study, we show that, compared with an age-matched and sex-matched control population, childhood-onset and adult-onset IgAV is associated with increased risk of hypertension and CKD. Adult-onset IgAV was not associated with IHD, cerebrovascular disease or VTE. 
VTE

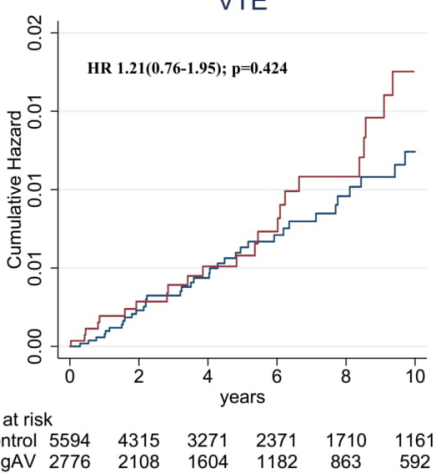

IHD

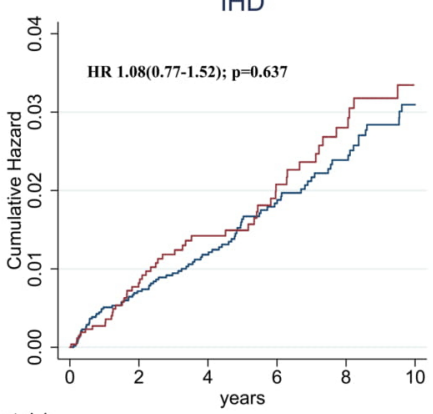

Number at risk

$\begin{array}{llllll}\text { Control } 5435 & 4172 & 3158 & 2272 & 1639 & 1106\end{array}$

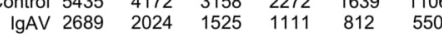

Stroke/TIA

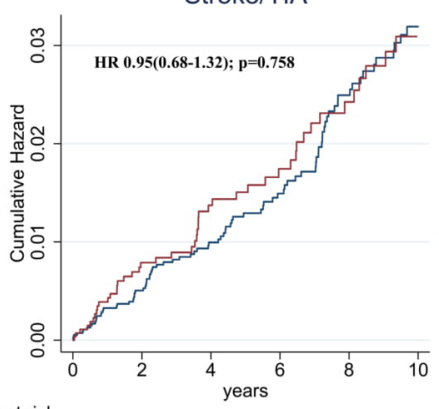

Number at risk

$\begin{array}{llllll}\text { Control 5548 } & 4278 & 3244 & 2344 & 1695 & 1144\end{array}$

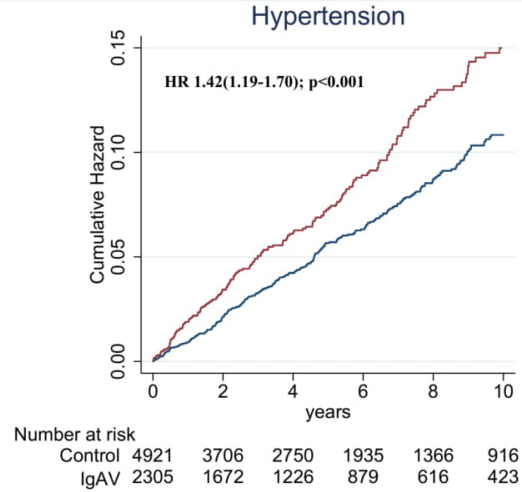

Mortality

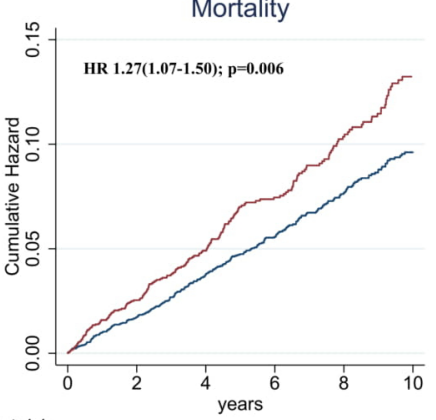

Number at risk

Control 5655 $4371 \quad 3321 \quad 2414 \quad 1751 \quad 1191$ $\begin{array}{llllll}\text { IgAV } 2828 & 2150 & 1635 & 1202 & 881 & 601\end{array}$

CKD

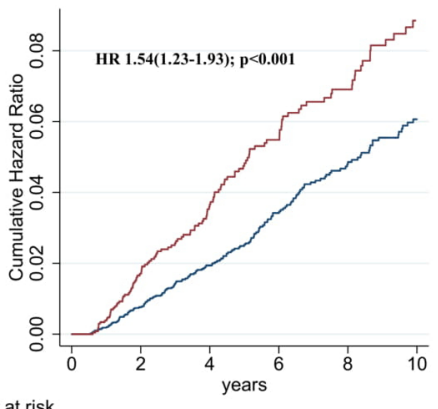

Number at risk

$\begin{array}{llllll}\text { Control 5410 } & 4149 & 3118 & 2229 & 1608 & 1090\end{array}$ $\begin{array}{rrrrrr}\text { IgAV } 2621 & 1962 & 1465 & 1064 & 772 & 526\end{array}$

Figure 2 Cumulative hazard curves for outcomes in adult-onset IgA vasculitis (IgAV). Cumulative hazard curves are displayed for each of the six outcomes under study in the adult-onset IgAV cohort. IHD, ischaemic heart disease; VTE, venous thromboembolism.

Table 3 Summary of primary outcomes in childhood-onset IgA vasculitis (IgAV) cases and corresponding controls

\begin{tabular}{|c|c|c|c|c|c|c|}
\hline & \multicolumn{2}{|l|}{ Hypertension } & \multicolumn{2}{|c|}{ Venous thromboembolism } & \multicolumn{2}{|c|}{ Chronic kidney disease } \\
\hline & $\operatorname{IgAV}$ & Control & $\lg A V$ & Control & $\operatorname{IgAV}$ & Control \\
\hline Patients, $\mathrm{n}$ & 10220 & 20574 & 10376 & 20773 & 10370 & 20763 \\
\hline Numbers of outcomes & 139 & 193 & 25 & 46 & 32 & 34 \\
\hline Person-years & 60753.91 & 123318.1 & 62333.31 & 125359.3 & 62185.31 & 125362.3 \\
\hline $\begin{array}{l}\text { Incidence rate (per } 1000 \text { person- } \\
\text { years) }\end{array}$ & 2.29 & 1.57 & 0.40 & 0.37 & 0.51 & 0.27 \\
\hline Adjusted HR (95\% Cl) & $1.52(1.22-1.89)$ & & $1.10(0.68-1.79)$ & & $1.89(1.16-3.07)$ & \\
\hline$P$ values & $<0.001$ & & 0.697 & & 0.010 & \\
\hline
\end{tabular}




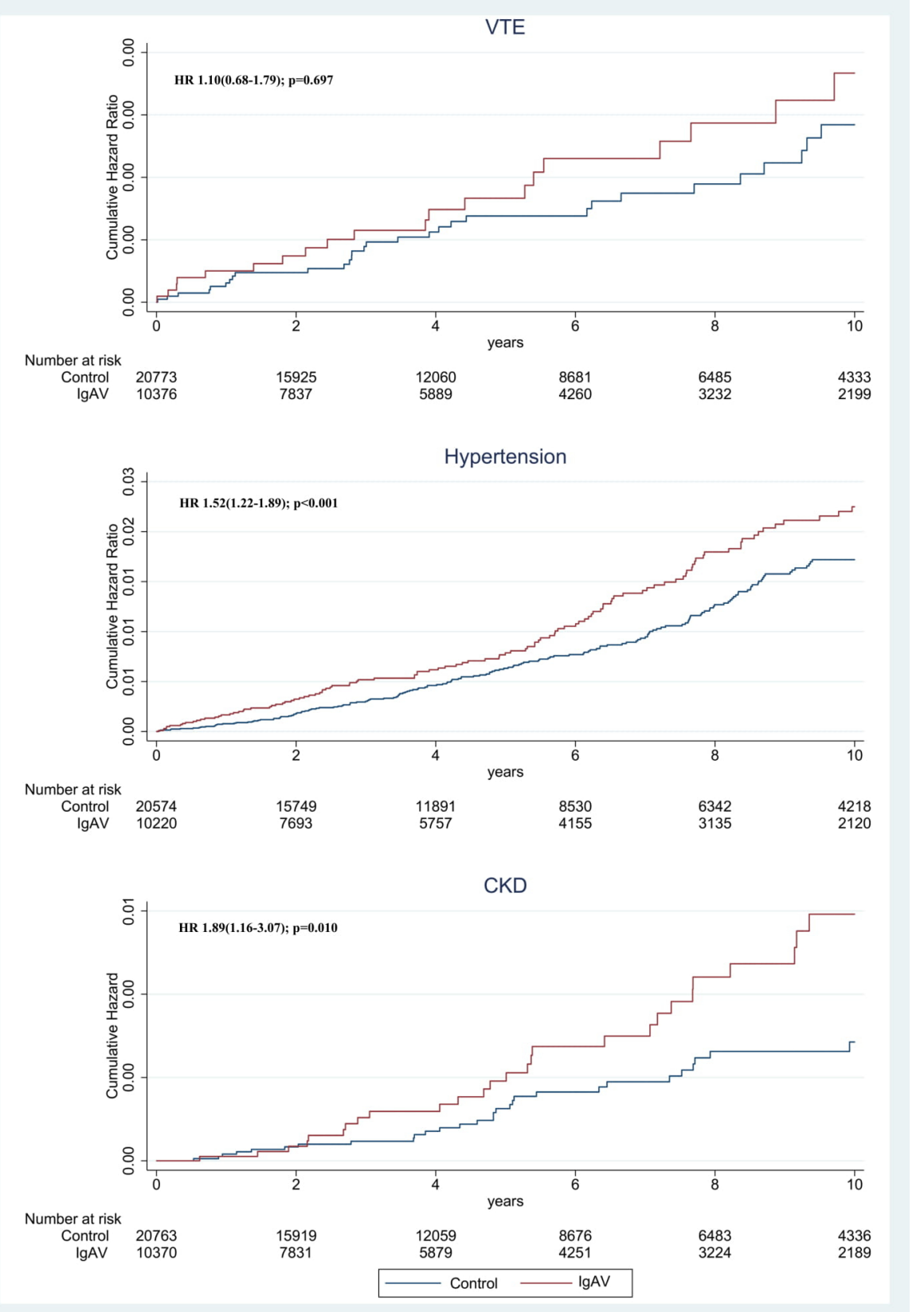

Figure 3 Cumulative hazard curves for outcomes in childhood-onset IgA vasculitis (IgAV). Cumulative hazard curves are displayed for each of the three outcomes under study in the childhood-onset IgAV cohort. VTE, venous thromboembolism.

Annual IgAV incidence from 2005 to 2016 in children (27.22 per 100000 person-years) and adults (2.20 per 100000 personyears) was marginally higher than previously reported (20 and $0.8-1.8$ per 100000 person-years in children and adults, respectively), likely due to the different case-finding strategies employed. ${ }^{23}$ Previous studies likely underestimated IgAV incidence as they had access to less comprehensive datasets. While incidence remained stable over the study period, prevalence of adult-onset and childhood-onset IgAV increased. This could be explained by improved documentation of existing IgAV diagnoses or may reflect increased patient survival. The former explanation is more likely given the short time period over which this increase occurred.

To our knowledge, this is the first controlled study examining incidence of IHD, stroke/TIA and VTE in adult patients with IgAV. Despite evidence from case series suggesting that
IgAV confers increased risk of these outcomes, ${ }^{8-19}$ we found no evidence of association between IgAV and IHD, stroke/TIA or VTE. We note that incidence of VTE was low in this cohort (1.67 per 1000 person-years). VTE may be an isolated acute event unrecorded in general practice records, and therefore this result should be interpreted with caution.

Although hypertension has been associated with poor outcomes in some patients with $\mathrm{IgAV}^{35}$ incidence of hypertension in childhood-onset and adult-onset IgAV was previously unknown. This study revealed similar risk for children and adults. The effect could be explained by renal manifestations of IgAV or by use of medications such as non-steroidal anti-inflammatory drugs and corticosteroids. The association remained significant in a sensitivity analysis using only hypertension recorded at least 1 year post-index date, suggesting that it is not solely an acute feature of the disease. 
IgAV is thought to have a poorer renal prognosis in adult patients than in children. ${ }^{5}{ }^{36}$ In patients with adult-onset IgAV, incidence of stage G3-5 CKD was 8.72 per 1000 personyears, with a 54\% increase in risk compared with controls. In patients with childhood-onset IgAV, incidence of CKD was much lower (0.51 per 1000 person-years) but with a similar HR. The increased incidence of CKD in adults compared with children may be explained by a higher burden of comorbidity and renal impairment at baseline.

A 35\% increase in all-cause mortality was observed in adultonset IgAV patients compared with controls. However, this effect was no longer statistically significant in a sensitivity analysis including only incident cases of IgAV. This may be explained by reduced length of follow-up when only incident cases are considered.

\section{Strengths and limitations}

Definition of exposure status depended on accurate coding of IgAV diagnosis in primary care medical records. These records do not include information on whether IgAV was diagnosed based solely on clinical criteria, or whether biopsy findings were used. We are unable to identify patients with renal involvement at diagnosis and recognise that there may be stronger associations with the outcomes if the cohort is restricted to patients with biopsy-proven IgAV, as previously shown in patients with biopsy-proven Henoch-Schönlein nephritis. ${ }^{37}$

Alternatively, other vasculitides, such as microscopic polyangiitis, may have been misdiagnosed as IgAV in the absence of histological investigation. Inclusion of such patients could increase the incidence of complications such as CKD. Although widespread testing for anti-neutrophil cytoplasm autoantibodies has been available since the 1990s, we have no data on anti-neutrophil cytoplasm antibody testing and cannot exclude that this diagnosis may have been missed in some cases.

It is possible that patients with greater disease severity were selectively coded in primary care, leading to overestimation of effect size. However, our incidence and prevalence estimates were similar, if not slightly higher than previously reported. Similarly, inaccurate coding of outcomes is a potential source of error. CKD is likely to be underdiagnosed in primary care, ${ }^{38}$ so practice records may underestimate its incidence. To minimise this risk, CKD was defined by eGFR criteria not clinical codes.

A further caveat is uncertainty regarding classification of IgAV as adult onset. In the primary analysis, all adult IgAV patients with coded date of diagnosis after their 16th birthday were included. However, some cases may have been inappropriately defined as adult onset, for example, if coded when the patient moved to a new practice. Nevertheless, results were replicated in sensitivity analyses using incident adult IgAV cases only, showing that our findings are robust to stricter definitions of adult-onset IgAV.

When considering cardiovascular outcomes in patients with adult-onset IgAV, it should be noted that the affected population was relatively young (mean age 43.3 years at study entry). Therefore, it is possible that length of follow-up in this study was insufficient to detect increased risk of IHD.

Finally, this study could be influenced by surveillance bias. Patients with IgAV may receive closer monitoring of blood pressure and renal function in primary care. This may contribute to the higher CCI scores observed in patients with adult-onset IgAV compared with controls.

Despite these limitations, a strength of this population-based study design is its external validity. Patients with IgAV and control participants were included from a primary care database which is broadly representative of the UK population in terms of ethnicity, chronic disease prevalence and mortality rates. ${ }^{23}$

\section{CONCLUSION}

This retrospective cohort study demonstrates associations between IgAV and hypertension and CKD. These findings emphasise the importance of blood pressure and renal function monitoring in patients with IgAV. Our data also suggest that IgAV should not be considered a 'single hit' disease, but that clinicians should monitor for long-term sequelae. Further research is required to clarify the cause of hypertension in patients with IgAV, and to investigate whether such patients suffer from additional long-term sequelae than that are currently unrecognised.

Contributors AT, LH and KN conceived the work. AT contributed to the analysis and interpretation of data, drafting the manuscript and revising for intellectual content. AS and KN contributed to the acquisition, analysis and interpretation of data, and drafting the manuscript. NJA contributed to data analysis and interpretation, and writing the manuscript. LH contributed to drafting the manuscript and revising for intellectual content. PC, CF and SB contributed to revising the manuscript for intellectual content. All authors approved the final version to be submitted for review.

Funding The authors have not declared a specific grant for this research from any funding agency in the public, commercial or not-for-profit sectors.

Competing interests None declared.

Patient consent Not required.

Ethics approval The THIN data collection scheme and research carried out using THIN data were approved by the NHS South-East Multicentre Research Ethics Committee in 2003; under the terms of this approval, studies must undergo independent scientific review. Approval for this analysis was obtained from the Scientific Review Committee (for the use of THIN data) in April 2018 (SRC reference number 18THIN016)

Provenance and peer review Not commissioned; externally peer reviewed.

\section{REFERENCES}

1 Jennette JC, Falk RJ, Bacon PA, et al. 2012 revised international chapel hill consensus conference nomenclature of vasculitides. Arthritis Rheum 2013;65:1-11.

2 Gardner-Medwin JM, Dolezalova P, Cummins C, et al. Incidence of Henoch-Schönlein purpura, Kawasaki disease, and rare vasculitides in children of different ethnic origins. Lancet 2002;360:1197-202

3 Piram M, Mahr A. Epidemiology of immunoglobulin A vasculitis (Henoch-Schönlein): current state of knowledge. Curr Opin Rheumatol 2013;25:171-8.

4 Heineke $\mathrm{MH}$, Ballering $\mathrm{AV}$, Jamin $\mathrm{A}$, et al. New insights in the pathogenesis of immunoglobulin A vasculitis (Henoch-Schönlein purpura). Autoimmun Rev 2017; 16:1246-53.

5 Blanco R, Martínez-Taboada VM, Rodríguez-Valverde V, et al. Henoch-Schönlein purpura in adulthood and childhood: two different expressions of the same syndrome. Arthritis Rheum 1997:40:859-64.

6 Kang Y, Park JS, Ha YJ, et al. Differences in clinical manifestations and outcomes between adult and child patients with Henoch-Schönlein purpura. J Korean Med Sci 2014;29:198-203.

7 Pillebout E, Verine J. Henoch-Schonlein purpura in the adult. La Rev Med interne 2014;35:372-81

8 Hayakawa K, Shiohara T. Two cases of Henoch-Schönlein purpura with transient myocardial ischaemia. Acta Derm Venereol 2003;83:393-4.

9 Eleftheriadis D. Severe coronary artery disease in the setting of Henoch-Schoenlein purpura. Int J Cardiol 2007;118:262-3.

10 Canpolat U, Yorgun H, Sahiner L, et al. Myocardial infarction due to coronary thrombosis in a patient with Henoch-Schönlein purpura. Herz 2012;37:801-3.

11 Veetil BM, Reed AM, Mattke AC. Coronary artery thickening with mucosal lesions in Henoch-Schönlein purpura. Pediatr Dermatol 2012;29:377-8.

12 Bellantoni A, Lo Presti P, Giordano A, et al. [A pediatric case of Schoenlein-Henoch purpura with clinical, serologic and electrocardiographic signs of myocardial damage]. G Ital Cardiol 2013; 14:622-5.

13 Zaidi AU, Berman B. Crossing the thrombotic threshold: deep vein thrombosis in Henoch-Schönlein purpura. Clin Pediatr 2014;53:1396-8.

14 Topaloglu R, Bayrakci US, Cil B, et al. Henoch-Schonlein purpura with high factor VIII levels and deep venous thrombosis: an association or coincidence? Rheumatol Int 2008;28:935-7.

15 Sari I, Akar S, Secil M, et al. Thrombosis and priapism in a patient with HenochSchonlein purpura. Rheumatol Int 2005:25:472-4. 
16 Diana A, Gaze H, Laubscher B, et al. A case of pediatric Henoch-Schönlein purpura and thrombosis of spermatic veins. J Pediatr Surg 2000;35:35.

17 Li L, Zhang J, Zhang Y, et al. Thrombosis warning in children suffering from henochschonlein purpura. Indian J Dermatol 2013;58:409.

18 Samanta SK, Mahapatra N, Aich B, et al. An unusual case of transient cortical blindness with sagittal sinus thrombosis in a case of Henoch-Schonlein purpura. Nepal J Ophthalmol 2012;4:333-5.

19 Abend NS, Licht DJ, Spencer CH. Lupus anticoagulant and thrombosis following Henoch-Schonlein purpura. Pediatr Neurol 2007;36:345-7.

20 Morgan MD, Turnbull J, Selamet U, et al. Increased incidence of cardiovascular events in patients with antineutrophil cytoplasmic antibody-associated vasculitides: a matched-pair cohort study. Arthritis Rheum 2009;60:3493-500.

21 Desbois A-C, Wechsler B, Cluzel P. [Cardiovascular involvement in Behcet'sdisease]. La Rev Med interne 2014;35:103-11.

22 Bramlage CP, Kröplin J, Wallbach M, et al. Management of cardiovascular risk factors in patients with ANCA-associated vasculitis. J Eval Clin Pract 2017;23:747-54.

23 Blak BT, Thompson M, Dattani H, et al. Generalisability of The Health Improvement Network (THIN) database: demographics, chronic disease prevalence and mortality rates. Inform Prim Care 2011;19:251-5.

24 Maguire A, Blak BT, Thompson M. The importance of defining periods of complete mortality reporting for research using automated data from primary care. Pharmacoepidemiol Drug Saf 2009;18:76-83.

25 Booth N. What are the Read Codes? Health Libr Rev 1994;11:177-82.

26 Denburg MR, Haynes K, Shults J, et al. Validation of The Health Improvement Network (THIN) database for epidemiologic studies of chronic kidney disease. Pharmacoepidemiol Drug Saf 2011;20:1138-49.

27 Lewis JD, Schinnar R, Bilker WB, et al. Validation studies of the health improvement network (THIN) database for pharmacoepidemiology research. Pharmacoepidemiol Drug Saf 2007;16:393-401.

28 Lee T, Lu N, Felson DT, et al. Use of non-steroidal anti-inflammatory drugs correlates with the risk of venous thromboembolism in knee osteoarthritis patients: a UK population-based case-control study. Rheumatology 2016;55:1099-105.
29 Lévesque LE, Hanley JA, Kezouh A, et al. Problem of immortal time bias in cohort studies: example using statins for preventing progression of diabetes. BMJ 2010;340:b5087.

30 Kidney Disease: Improving Global Outcomes(KDIGO) CKD Work Group. KDIGO 2012 Clinical Practice Guideline for the Evaluation and Management of Chronic Kidney Disease. Kidney Int Supp/ 2013;3:4.

31 Daly B, Toulis KA, Thomas N, et al. Increased risk of ischemic heart disease, hypertension, and type 2 diabetes in women with previous gestational diabetes mellitus, a target group in general practice for preventive interventions: A population-based cohort study. PLoS Med 2018;15:e1002488.

32 Chandan IS, Thomas T, Lee $S$, et al. The association between idiopathic thrombocytopenic purpura and cardiovascular disease: a retrospective cohort study. J Thromb Haemost 2018;16:474-80.

33 Adams J, Ryan V, White M. How accurate are Townsend Deprivation Scores as predictors of self-reported health? A comparison with individual level data. J Public Health 2005;27:101-6.

34 Charlson ME, Pompei P, Ales KL, et al. A new method of classifying prognostic comorbidity in longitudinal studies: development and validation. J Chronic Dis 1987:40:373-83.

35 Shrestha S, Sumingan N, Tan J, et al. Henoch Schönlein purpura with nephritis in adults: adverse prognostic indicators in a UK population. QJM 2006;99:253-65.

36 Hung SP, Yang YH, Lin YT, et al. Clinical manifestations and outcomes of HenochSchönlein purpura: comparison between adults and children. Pediatr Neonatol 2009:50:162-8.

37 Pillebout E, Thervet E, Hill G, et al. Henoch-Schönlein Purpura in adults: outcome and prognostic factors. J Am Soc Nephrol 2002;13:1271-8.

38 Jain $\mathrm{P}$, Calvert M, Cockwell $\mathrm{P}$, et al. The need for improved identification and accurate classification of stages 3-5 Chronic Kidney Disease in primary care: retrospective cohort study. PLoS One 2014;9:e100831. 